\title{
Soft Compactness and Soft Topological Separate axioms
}

\author{
Li Fu${ }^{1}$, Hua Fu ${ }^{2}$ \\ 1.School of Mathematics and Statistics, Qinghai Nationalities university Xining, Qinghai 810000, P.R.China; \\ 2. Fujian Police College, Fujian, Fuzhou, 350008, P.R.China
}

\begin{abstract}
In this paper, we further discuss soft compactness and soft separation axioms in the soft topological space over the rough soft formal context $\mathrm{T}=(G, M, R, F)$. We define the compact soft topological space, give soft separation axioms of soft rough topological space and study their relationship in the soft topological space over the rough soft formal context.
\end{abstract}

\section{Keywords}

rough soft formal context, soft rough topological space, soft open(closed) set, countable(finite) cover, compactness.

\section{Introduction}

Topology Euler, $M$ can be formally defined as the study of qualitative properties of certain objects (called topological spaces) that are invariant under a certain kind of transformation (called a continuous map). Topology has been become one of the great unifying ideas of mathematics, and topology can be applied to many field, for example, when the fundamental concept of a fuzzy set was introduced by Zadeh in $1965 \mathrm{~L}$, Chang $C$ introduced fuzzy topological spaces in 1968. Later on, Many researchers studied and developed it, such as, author replaced the closed interval by a more general lattice $A$. Ying ying studied Hohles topology and called it fuzzifying topology.

The uncertainty of data modeling the problems in engineering, physics, computer sciences, economics, social sciences, medical science and many other diverse fields. And formal concept context Wille, ganter , rough set Pawlak, z, kent, soft set molodsov, $p$ are extensively applied in the uncertainty reasoning.

Shabir and Naz Shbir launched the study of soft topological spaces which was defined over an initial universe with a fixed set of parameters, and gave the concepts including soft open(closed)set, soft interior points and so on, they defined and discussed the soft $T_{i}$-space .Authors Naim defined soft topology on a soft set, and presented its related properties. Authors Sabir continued investigating the properties of soft topological spaces. Some authors $z h$ discussed the relationship among soft sets, soft rough sets and topologies.

Author $l i$ defined the rough soft formal context based on rough formal context $f u$, and discussed the rough properties of rough formal context in soft set. Author $f l$ defined some topological operators in the rough soft formal context, defined the soft rough topological space, and some topological properties were discussed over the soft rough topological space. Authors $f l i$ discussed the soft topological separation axioms in the rough soft formal context.

In this paper, we continue to study the soft rough topology, and we discuss soft compactness and soft separation axioms in the soft topological space over the rough soft formal context. The rest of this paper is organized as following. In section 2, we review some basic concepts and properties of rough concept formal soft sets, and soft rough topology, soft rough topological space over the rough soft formal context $T=(G, M, R, F)$. In section 3 , we define the compact soft topological space, give soft separation axioms of soft rough topological space and study the relationship between compactness and separation axioms in the soft topological space over the rough soft formal context $T=(G, M, R, F)$. Conclusions are given in section 4.

\section{Basic knowledge}

Definition 2.1 $M$ Let $\AA$ be a collection of sets, be a set, if $B \subseteq \bigcup_{A \in A} A$, then $\AA$ is a cover of $B$, if $\AA$ is a countable class or a finite class, then $\AA$ is a countable cover or a finite cover of $B$; if $\AA_{1} \subseteq \AA$, and $\AA_{1}$ is a cover of $B$ , then $\AA_{1}$ is a sub-cover of $\AA$ for $B$. 
Definition 2.2 molodsov Let $U$ be an initial universe set and $E$ be a set of parameters. Let $\wp(U)$ denotes the power set of $U$ and $A \subset E$. Then a pair $(F, A)$ is called a soft set over $U$, where $F: A \rightarrow \wp(U)$ is a mapping.

That is, the soft set is a parameterized family of subsets of the set $U$. Every set $F(e), \forall e \in E$, from this family may be considered as the set of e-elements of the soft set $(F, E)$, or considered as the set of e-approximate elements of the soft set.

Definition $2.3 \mathrm{li}$ Let $(G, M, R)$ is a rough formal context, $G$ is objects set, is also called the universe, $M$ is attributes set. A pair $(F, B)$ is a soft set over $G$, where and $F: B \rightarrow \wp(U)$ is a set-value mapping over $G$, furthermore, the lower and upper rough approximations of pair $(F, B)$ are denoted by

$$
\underline{R}(F, B)=(\bar{F}, B), \bar{R}(F, B)=(\bar{F}, B),
$$

which are soft sets over $G$ with the set-valued mappings given by $\underline{F}(x)=\underline{B}(F(x))$ and $\bar{F}(x)=\bar{B}(F(x))$, where $x \in B$. The operators $\bar{R}, \underline{R}$ are called the lower and upper rough approximation operators on soft set $(F, B)$.

If $\bar{R}=\underline{R}$,we say that the soft set $(F, B)$ is definable, otherwise, $(F, B)$ is rough.

we call such quadruple tuple $(G, M, R, F)$ as rough soft formal context, and, such soft set $(F, B)$ on the rough soft formal context $(G, M, R, F)$ which is called rough soft formal set.

Obviously, $\forall x \in B \subseteq M, F(x) \subseteq G$ is a parameterized family of subsets of $G$, and $F(x)$ is the set of $x$-approximate elements in $(G, M, R, F)$.

Definition $2.4 l i$ Let $(G, M, R, F)$ be a rough soft formal context with the objects set $G$, and attributes set $M$. $B_{1}, B_{2} \subseteq M,\left(F, B_{1}\right)$ and $\left(F_{1}, B_{2}\right)$ are two soft sets over $G$ on the rough soft formal context $(G, M, R, F)$. $F_{1}: B \rightarrow \wp(G)$ is a set-value mapping over the rough soft formal context $(G, M, R, F)$.

(i) If $B_{1} \subseteq B_{2}$, and $\forall x \in B_{1} \subseteq B_{2}$, having $F(x) \subseteq F_{1}(x)$, then the soft sets $\left(F, B_{1}\right)$ is a soft subset of the soft set $\left(F_{1}, B_{2}\right)$, denoted as $\left(F, B_{1}\right) \widetilde{\subset}\left(F_{1}, B_{2}\right)$.

(ii) Two soft sets $\left(F, B_{1}\right)$ and $\left(F_{1}, B_{2}\right)$ on the rough soft formal context $(G, M, R, F)$ are said soft equal, if $\left(F, B_{1}\right) \widetilde{\subset}\left(F_{1}, B_{2}\right)$, and $\left(F_{1}, B_{1}\right) \simeq\left(F, B_{2}\right)$. We simply denote by $\left(F, B_{1}\right)=\left(F_{1}, B_{2}\right)$.

(iii) The relative complement of $(F, B)$ is denoted by $(F, B)^{c}$ and is defined by $(F, B)^{c}=\left(F^{c}, B\right)$, where $F^{c}: B \rightarrow \wp(G)$, and $F^{c}(x)=G-F(x), \forall x \in B$.

Clearly, $\left((F, B)^{c}\right)^{c}=(F, B)$.

(iv) $(F, B)$ is said to be a relative null rough soft formal set denoted by $\mathcal{N}$, if $\forall x \in B, F(x)=\varnothing$; if $B=M$, then is called absolute null rough soft formal set, denoted as $\widetilde{\emptyset}$.

(v) $(F, B)$ is said to be a relative whole rough soft formal set denoted by $\tilde{G}$, if $\forall x \in B, F(x)=G$.

Definition $2.5 \mathrm{li}$ Let $(G, M, R, F)$ be the rough soft formal context, $\left(F_{1}, B_{1}\right)$ and $\left(F_{2}, B_{2}\right)$ are two rough soft formal sets over $(G, M, R, F)$, in which $F_{1}, F_{2}: B \rightarrow \wp(G)$ are two set-value mappings.

(i) The union $\left(F_{1}, B_{1}\right)$ and $\left(F_{2}, B_{2}\right)$ is the rough soft formal set $(H, C)$, where $C=B_{1} \cup B_{2}$, and $\forall e \in C$, denoted as 
$\left(F_{1}, B_{1}\right) \tilde{U}\left(F_{2}, B_{2}\right)=(H, C)=\left(H, B_{1} \cup B_{2}\right)$ where

(ii) The intersection of $\left(F_{1}, B_{1}\right)$ and $\left(F_{2}, B_{2}\right)$ is the soft rough formal set $(H, C)$ is denoted as $\left(F_{1}, B_{1}\right) \cap\left(F_{2}, B_{2}\right)$ and is defined as $\left(F_{1}, B_{1}\right) \sqcap\left(F_{2}, B_{2}\right)=(H, C)$, where $C=B_{1} \cap B_{2}$, and $\forall e \in C, H(e)=F_{1}(e) \cap F_{2}(e)$.

Definition 2.6 $f l$ Let $T=(G, M, R, F)$ be a rough soft formal context over the object set $G$ and attributes set $M, B_{i} \subseteq M$ which is the collection of soft sets on the rough soft formal context $(G, M, R, F)$, if it is satisfied:

(1) $\widetilde{\emptyset} \in \tau, \tilde{G} \in \tau$

(2) The union of any number of soft sets in $\tau$ belongs to $\tau$, that is, $\tau$ is closed for the any union of soft sets over $T$.

(3) The intersection of any two soft sets in $\tau$ belongs to $\tau$, that is, $\tau$ is closed for the finite intersection of soft sets over $T$. Then the collection $\tau$ is called a soft topology over the rough soft formal context $T$ (simply called soft rough topology). The triplet $(G, \tau, M)$ is called a soft topological space over the rough soft formal context $T$ (simply called soft rough topological space).

The members of $\tau$ are soft open sets in $T$, the relative complement $(F, B)^{c}=\left(F^{c}, B\right)$ is said to be a soft closed set in $T$ if $(F, B)^{c} \in \tau$.

\section{The soft compact topological space and soft topological separate axioms}

In the following, we study based on the rough soft formal context $T=(G, M, R, F)$ in which $G$ is an initial universe set $M$ is a set of attributes, $(G, \tau, M)$ be a soft topological space over $T$ set, and $(F, B)$ is a soft set over $T$.

Definition 3.1 Let $(G, \tau, M)$ be a soft topological space over $T,(F, B)$ be a soft set over $G$, and $x \in G$. we say $x \in(F, B)$ and read as $x$ belongs to the soft set $(F, B)$, if $x \in F(e)$ for all $e \in B$ and if there is some $e \in B$, such that $x \notin F(e)$, then $x \notin(F, B)$, read as $x$ does not belong to the soft set $(F, B)$.

Definition 3.2 Let $(G, \tau, M)$ be a soft topological space over $T, G$ is an initial universe set, $M$ is a set of attributes, and $(F, B)$ is a soft set over $G, x \in G$, then:

(i) soft interior of soft set $(F, B)$ over $G$ is denoted by $(F, B)^{\circ}$ and is defined as the union of all soft open set contained in $(F, B)$, that is, $(F, B)^{\circ}=\tilde{\bigcup}\left\{\left(F_{i}, B_{i}\right) \widetilde{\subset}(F, B) \in \tau\right\}$. Such $(F, B)^{\circ}$ is the largest soft open set contained in of $(F, B)$;

(ii) The soft closure of $(F, B)$, denoted by $\overline{(F, B)}$ is the intersection of all soft closed super set of soft set $(F, B)$, that is $\overline{(F, B)}=\bigcap\left\{\left(F_{i}, B_{i}\right) \mid(F, B) \subseteq\left(F_{i}, B_{i}\right) \in \tau\right\}$. Clearly, $\overline{(F, B)}$ is the smallest soft closed set over $T$ which contain the soft set $(F, B)$;

(iii) If there is a soft open set $\left(F_{1}, B_{1}\right)$ over $G$, such that $x \in\left(F_{1}, B_{1}\right) \tilde{\subset}(F, B)$, then $x$ is a soft interior point of $(F, B)$ and $(F, B)$ is the soft neighborhood of $x$.

Similarly, $\left(F_{1}, B_{1}\right) ;(F, B)$ are two soft sets over $G$, if $\left(F_{1}, B_{1}\right) \tilde{\subset}(F, B)^{\circ}$, then $(F, B)$ is the soft 
neighborhood of $\left(F_{1}, B_{1}\right)$.

Specially, if $(F, B)$ is a soft open (closed) set, then $(F, B)$ is a soft open (closed) neighborhood of $x$.

Definition 3.3 Let $(G, \tau, M)$ be a soft topological space over $T,(F, B)$ be a soft set over $G$, and the collection of soft sets $\AA=\{(F, B) \mid(F, B)$ is soft open (closed), $(F, B) \in \tau\}$ is a cover of $G$, then $A$ is a soft open(closed) cover of $G$.

Definition 3.4 Let $(G, \tau, M)$ be a soft topological space over $G$, is the collection soft open sets over $G$, and $x, y \in G$ and $x \neq y$, if there exist soft neighborhood $\left(F_{1}, M\right)$ of $x$ and soft neighborhood $\left(F_{2}, M\right)$ of $y$ such that $y \notin\left(F_{1}, M\right)$ or $x \notin\left(F_{2}, M\right)$, that is, for any $x \neq y \in G$, there exist the soft open sets $\left(F_{1}, M\right),\left(F_{2}, M\right) \in \tau$, such that either

$$
\begin{gathered}
\text { (i) } x \in\left(F_{1}, M\right) \text { and } y \notin\left(F_{1}, M\right) \text { or } \\
\text { (ii) } y \in\left(F_{2}, M\right) \text { and } x \notin\left(F_{2}, M\right) \text {, }
\end{gathered}
$$

then soft rough topological space $(G, \tau, M)$ over $G$ is called a soft rough $T_{0}$-space.

Obviously, if $(G, \tau, M)$ is a soft rough topological space, in which $\tau$ is a trivial soft rough topology over $G$, and $|G| \geq 2,|G|$ represents the cardinality of $G$, that is, the numbers of $G$. Then $(G, \tau, M)$ over $G$ is not a soft rough $T_{0}$-space.

Definition 3.5 Let $(G, \tau, M)$ be a soft topological space over $G$, is the collection soft open sets over $G$, and $x, y \in G$ and $x \neq y$, if there exist soft neighborhood $\left(F_{1}, M\right)$ of $x$ and soft neighborhood $\left(F_{2}, M\right)$ of $y$ such that $y \notin\left(F_{1}, M\right)$, that is, for any $x \neq y \in G$, there exist the soft open sets $\left(F_{1}, M\right),\left(F_{2}, M\right) \in \tau$, such that $x \in\left(F_{1}, M\right), y \notin\left(F_{1}, M\right)$ and $y \in\left(F_{2}, M\right), x \notin\left(F_{2}, M\right)$, then soft topological space $(G, \tau, M)$ over $G$ is called a soft rough $T_{1}$-space. not hold.

Obviously, if the triplet $(G, \tau, M)$ is a soft rough $T_{1}$-space, then it is also a $T_{0}$-space, however, the converse does

Definition 3. 6 Let $(G, \tau, M)$ be a soft topological space over $G$, is the collection soft open sets over $G$, and $x, y \in G$ and $x \neq y$, if there exist soft neighborhood $\left(F_{1}, M\right)$ of $x$ and soft neighborhood $\left(F_{2}, M\right)$ of $y$ such that $y \notin\left(F_{1}, M\right), x \notin\left(F_{2}, M\right)$, and $\left(F_{1}, B_{1}\right) \sqcap\left(F_{2}, B_{2}\right)=\widetilde{\varnothing}$, then soft topological space $(G, \tau, M)$ over $G$ is called a soft rough $T_{2}$-space, which is also called a soft rough Hausdröff space.

Definition 3.7 Let the triplet $(G, \tau, M)$ be a soft topological space over $G$, for all $x \in G$ and $(F, M)$ is a soft closed set in $T$, if there exist two soft open sets $\left(F_{1}, M\right),\left(F_{2}, M\right)$ in $\tau$, such that $x \in\left(F_{1}, M\right)$, and $(F, M) \widetilde{\subset}\left(F_{2}, M\right)$, and $\left(F_{1}, M\right) \sqcap\left(F_{2}, M\right)=\widetilde{\emptyset}$, then soft topological space $(G, \tau, M)$ is called a soft rough regular space.

Definition 3.8 Let the triplet $(G, \tau, M)$ be a soft topological space over $G,(G, \tau, M)$ be a soft rough topological space over $G,\left(F_{1}, M\right),\left(F_{2}, M\right)$ are soft closed sets in $\tau$, if there exist soft open sets $\left(F_{3}, M\right),\left(F_{4}, M\right)$ in $\tau$ such that $\left(F_{1}, M\right) \widetilde{\subset}\left(F_{3}, M\right),\left(F_{2}, M\right) \widetilde{\subset}\left(F_{4}, M\right)$, and $\left(F_{3}, M\right) \sqcap\left(F_{4}, M\right)=\widetilde{\emptyset}$, then soft topological space 
$(G, T, M)$ is called a soft rough normal space.

Definition 3.9 Let $(G, \tau, M)$ be a soft topological space over $T,(F, B)$ be a soft set over $T$, and the collection of soft sets $\stackrel{\circ}{A}=\{(F, B) \mid(F, B)$ is an soft open set in $\tau\}$ be any soft open cover of $G$, if $A$ has a soft countable subcover of $G$, then $(G, \tau, M)$ is a soft Lindelöff topological space over $T$.

Obviously, let $(G, \tau, M)$ be a discrete soft topological space over $\mathrm{T}$, and $(F, B)$ be a soft set over $G$, suppose that the soft topology $\tau=\left\{\left(F_{i}, M\right) \mid F_{i}: M \rightarrow \wp(G)\right\}$ is a discrete soft rough topology over $\mathrm{T}$. Then $(G, \tau, M)$ is not a soft Lindel $\ddot{O}$ ff topological space over $\mathrm{T}$.

Definition 3.10 Let $(G, \tau, M)$ be a soft topological space over $T,(F, B)$ is a soft set over $T$, and the collection of soft sets $\stackrel{\circ}{A}=\{(F, B) \mid(F, B)$ is an soft open set in $\tau\}$ be a soft open cover of $G$, if any soft open cover of $G$ has a soft finite sub-cover, then $(G, \tau, M)$ is a compact soft topological space over $T$.

Clearly, if $(G, \tau, M)$ is a compact soft topological space over $T$, then it must be a Lindelöff soft topological space over $T$, however, the converse does not hold.

Proposition 3.1 Let $(G, \tau, M)$ be a soft topological space over $T$, if $(F, B)$ is any finite soft subset of $\tilde{G}$, then $(F, B)$ is a compact soft subset of $\tilde{G}$.

Proof Let $(G, \tau, M)$ be a soft topological space over $T$, and $(F, B)$ be any finite soft subset of $\tilde{G}$, the collection $A=\{(F, B) \mid(F, B)$ is soft set in $T,(F, B) \tilde{\subset} \tilde{G}\}$ covers $(F, B)$, for all $x \in(F, B)$, that is, $x \in F(e)$ for all $e \in B$, taking $\dot{A}_{1}=\{(F, B) \mid(F, B)$ is soft set in $T$, and $x \in(F, B)\}$, then $\AA_{1}$ is a finite sub-cover of $(F, B)$. Hence, $(F, B)$ is a compact soft subset of $\tilde{G}$.

Proposition 3.2 Let $\left(G, \tau_{1}, M\right),\left(G, \tau_{2}, M\right)$ be two soft topological spaces over $T, \tau_{1} \subseteq \tau_{2}$, and $\left(G, \tau_{2}, M\right)$ be a compact soft topological spaces over $T$, then $\left(G, \tau_{1}, M\right)$ is also a compact soft topological spaces over $T$.

Proof: Let $\left(G, \tau_{1}, M\right),\left(G, \tau_{2}, M\right)$ be two soft topological spaces over $T$. Suppose the collection $\AA=\{(F, B) \mid(F, B)$ is a soft set in $T,(F, B) \widetilde{\subset} \widetilde{\mathrm{G}}\}$ which is an open cover of $\tau_{1}$, by $\tau_{1} \subseteq \tau_{2}$ having the collection $\AA$ is also an open cover of $\tau_{2}$, and $\left(G, \tau_{2}, M\right)$ be a compact soft topological spaces over $T$, then there exist a finite sub-cover $\AA_{1}$ of $\stackrel{\circ}{A}$ which is a finite open cover of $\tau_{1}$.

Hence, $\left(G, \tau_{1}, M\right)$ is also a compact soft topological spaces over $T$.

Proposition 3.3 Let $(G, \tau, M)$ be a soft topological space over $T$ if $\left(F_{1}, B_{1}\right),\left(F_{2}, B_{2}\right), \cdots,\left(F_{n}, F_{n}\right)$ are compact soft subsets of $G$, then $\left(F_{1}, B_{1}\right) \tilde{U}\left(F_{2}, B_{2}\right) \tilde{U} \cdots \tilde{U}\left(F_{n}, F_{n}\right)$ is a compact soft subset of $G$.

Proof: Let $(G, \tau, M)$ be a soft topological space over $T$ and $\left(F_{1}, B_{1}\right),\left(F_{2}, B_{2}\right), \cdots,\left(F_{n}, B_{n}\right)$ be compact soft subsets of $\tilde{G}$. Assume $U=\bigcup_{i=1}^{n}\left(F_{i}, B_{i}\right)$ and the collection $\stackrel{\circ}{A}=\{(F, B) \mid(F, B)$ is a soft $\operatorname{set}$ in $T,(F, B) \tilde{\subset} \widetilde{\mathrm{G}}\}$ which is 
any open cover of $U$, then the collection is also an open cover of $\left(F_{i}, B_{i}\right), i=1,2, \cdots, n$, by $\left(F_{i}, B_{i}\right)$ being a compact soft subset of $\tilde{G}$, so there exists a sub-collection $\stackrel{\circ}{A}_{i}$ of $\stackrel{\circ}{A}$ which is sub-cover of $\left(F_{i}, B_{i}\right), i=1,2, \cdots, n$, and $\bigcap_{i=1}^{n} \dot{\circ}_{i}$ is a finite cover of $U$. Hence, $U=\bigcup_{i=1}^{n}\left(F_{i}, B_{i}\right)$ is a compact soft subset of $\tilde{G}$.

Similarly, we have the following property:

Proposition 3.4 Let $(G, \tau, M)$ be a soft topological space over $T$, the collection $\stackrel{\circ}{A}=\{(F, B) \mid(F, B)$ is a compact soft closed subset of $\tilde{\mathrm{G}}\}$, then $\bigcap_{(F, B) \in A}(F, B)$ is a compact soft subset of $\tilde{G}$

Proof Let $(G, \tau, M)$ be a soft topological space over $T$, and $\stackrel{\circ}{A}=\{(F, B) \mid(F, B)$ is a compact soft closed subset of $\tilde{\mathrm{G}}\}, \quad$ then $\Pi_{(F, B) \in A}(F, B)$ is a soft closed subset of $\tilde{G}$.and $\forall(F, B) \in T, \sqcap \underset{(F, B) \in A}{(}(F, B) \tilde{\subset}(F, B)$. Hence, $\Pi_{(F, B) \in A}(F, B)$ is a compact soft subset of $\tilde{G}$.

Furthermore, in soft Hausdröff space, we have:

Furthermore, in soft Hausdr $\ddot{O}$ ff space, we have:

Proposition 3.5 Let $(G, \tau, M)$ be a soft Hausdröff space over $T$, the collection $\stackrel{\circ}{A}=\{(F, B) \mid(F, B)$ is a compact soft closed subset of $\widetilde{G}\}$, then $\Pi_{(F, B) \in A}(F, B)$ is a compact soft subset of $\tilde{G}$. Proof: Let $(G, \tau, M)$ be a soft Hausdröff space over $T$, and $\stackrel{\circ}{A}=\{(F, B) \mid(F, B)$ is a compact soft closed subset of $\tilde{\mathrm{G}}\}$, then $\Pi_{(F, B) \in \dot{A}}(F, B)$ is a soft closed subset of $\tilde{G}$.and $\forall(F, B) \in T, \Pi \underset{(F, B) \in A}{(}(F, B) \tilde{\subset}(F, B)$. Hence, $\quad \Pi_{(F, B) \in A}(F, B)$ is a compact soft subset of $\tilde{G}$.

Theorem 3.1 Let $(G, \tau, M)$ be a soft Hausdröff space over $T$, the collection $\stackrel{\circ}{A}=\{(F, B) \mid(F, B)$ is a compact soft closed subset of $\tilde{\mathrm{G}}\}$, then $\Pi_{(F, B) \in A}(F, B)$ is a compact soft subset of $\tilde{G}$.

Proof: Suppose that $A$ is a compact subset of $\tilde{G}$, and $x \notin A, \forall y \in A$, by $(G, \tau, M)$ being a soft Hausdröff space over $T$, then there are open neighborhoods $U_{x}$ of $x$ and $V_{x}$ of $y$, such that $U_{x} \sqcap V_{x}=\widetilde{\emptyset}$.

The collection $\left\{V_{y} \mid y \in A\right\}$ is an open cover of compact subset $A$, which has a finite sub-collection, assume that $\left\{V_{y_{1}}, V_{y_{2}}, \cdots V_{y_{n}}\right\}$ covers $A$, and let $U=\bigcap_{i=1}^{n} U_{y_{i}}, V=\bigcap_{i=1}^{n} V_{y_{i}}$ be the open neighborhood of $x$ and $A$, respectively, and for all $i=1,2, \cdots, n$, having $U \sqcap V_{y_{i}}=U_{y_{1}} \sqcap U_{y_{2}} \sqcap \cdots U_{y_{n}} \sqcap V_{y_{i}}=\widetilde{\varnothing}$.

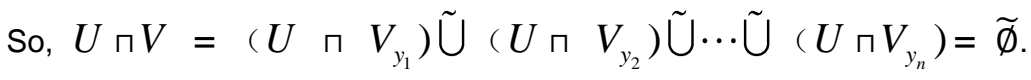

Corollary 3.1 Every compact subset of soft Hausdröff space over $T$ is a soft closed set. 
Proof Let $(G, \tau, M)$ be a soft Hausdröff space over $T$, and $(F, B)$ is a compact soft subset of $\tilde{G}_{\text {。 }}$ $\forall x \in G, x \notin(F, B)$, then $x \notin U \sqcap(\tilde{G}-\{x\})$, in which, $U$ is any open soft neighborhood of $(F, B)$, so, $(F, B)$ is a soft closed set.

Corollary 3.2 Let $(G, \tau, M)$ be a soft soft Hausdröff space over $T$, and $(F, B)$ be a soft closed set if and only if $(F, B)$ is a compact subset of $\tilde{G}$.

We have

Compact soft topological space: closed soft set $\Rightarrow$ compact soft subset soft Hausdorff space: closed soft set $\Leftarrow$ compact soft subset

Compact soft Hausdorff space: closed soft set $\Leftrightarrow$ compact soft subset

Corollary 3.3 Every compact soft Hausdröff space over $T$ is a regular soft topological space.

Proof Let $(G, \tau, M)$ be soft Hausdröff space over $T$, and $(F, B)$ be a soft closed set over $(G, \tau, M)$. For all $x \in G, x \notin(F, B)$, then $(F, B)$ is a compact soft subset, and there exist open neighborhoods $U$ ans $V$ of $x$ and $(F, B)$, respectively, such that $U \sqcap V=\varnothing$, that is , $(G, \tau, M)$ is a regular soft topological space over $T$.

Theorem 3.2 Let $(G, \tau, M)$ be a soft Hausdröff space over $T$, and $\left(F_{1}, B_{1}\right),\left(F_{2}, B_{2}\right)$ be compact subsets of $\tilde{G}$, and $\left(F_{1}, B_{1}\right) \sqcap\left(F_{2}, B_{2}\right)=\widetilde{\emptyset}$, then there exist soft open neighbors $U$ and $V$ of $\left(F_{1}, B_{1}\right)$ and $\left(F_{2}, B_{2}\right)$, such that $U \sqcap V=\widetilde{\varnothing}$

Proof Suppose that $\left(F_{1}, B_{1}\right),\left(F_{2}, B_{2}\right)$ are compact subsets of $\tilde{G}$, and $\left(F_{1}, B_{1}\right)^{\Pi}\left(F_{2}, B_{2}\right)=\widetilde{\emptyset}$. For all $x \in\left(F_{1}, B_{1}\right)$, there exists an open soft neighborhood $U_{x}$ and $V_{x}$ of $x$ and $\left(F_{2}, B_{2}\right)$, such that $U_{x}{ }^{\sqcap} V_{x}=\widetilde{\varnothing}$, and the collection $A=\left\{U_{x} \mid x \in\left(F_{1}, B_{1}\right),\left(F_{1}, B_{1}\right) \in T\right\}$ is an open cover of $\left(F_{1}, B_{1}\right)$,then there exists a finite sub-collection $\left\{U_{x_{1}}, U_{x_{2}}, \cdots, U_{x_{n}}\right\}$ of $U_{x}$ which covers $\left(F_{1}, B_{1}\right)$, similarly, the finite sub-collection $\left\{V_{x_{1}}, V_{x_{2}}, \cdots, V_{x_{n}}\right\}$ of $V_{x}$ which covers $\left(F_{2}, B_{2}\right)$, Assume $U=\bigcup_{i=1}^{n} U_{x_{i}}, V=\bigcup_{i=1}^{n} V_{x_{i}}$, then $U_{x_{i}}{ }^{\Pi} V=\widetilde{\emptyset}$, so $U \sqcap V=\widetilde{\emptyset}$.

Proposition 3.5 Let $(G, \tau, M)$ be a soft regular topological space over $T$, and $(F, B)$ be a compact subset of $\tilde{G}$ ,and $\left(F_{1}, B_{1}\right) \tilde{\subset} \tilde{G}$, If $(F, B) \tilde{\subset}\left(F_{1}, B_{1}\right) \tilde{\subset} \overline{(F, B)}$,then $\left(F_{1}, B_{1}\right)$ is a compact subset of $\tilde{G}$.

Proof Suppose that the collection $\stackrel{\circ}{A}$ of soft set in $\tau$ is an open cover of $\left(F_{1}, B_{1}\right)$, by $(F, B) \tilde{\subset}\left(F_{1}, B_{1}\right) \tilde{\subset} \overline{(F, B)}$, and $(F, B)$ be a compact subset of $\tilde{G}$, having the collection $A$ is also an open cover of $(F, B)$, so there exists a finite sub-collection $A_{1}$ of $A$ which is a finite cover of $(F, B)$, and by $(G, T, M)$ being a soft regular topological space over $T$ ,then there is an open soft neighborhood $V$ of $(F, B)$, such that $V \tilde{\subset} \tilde{U}_{U \in A_{1}} U$, and $\left(F_{1}, B_{1}\right) \tilde{\subset} \overline{(F, B)} \tilde{\subset V \tilde{\subset} \tilde{U}}{ }_{U \in A_{1}} U$, that is $A_{1}$ is a finite open cover of $\left(F_{1}, B_{1}\right)$. Hence, $\left(F_{1}, B_{1}\right)$ is a compact subset of $\tilde{G}$. 
Proposition 3.6 Let $(G, \tau, M)$ be a soft regular topological space over $T$, and $(F, B)$ be a compact subset of $\tilde{G}$ . $U$ is an open soft neighborhood of $(F, B)$, then there is an open soft neighborhood $V$ of $(F, B)$ such that $\bar{V} \tilde{\subset} U$.

Proof: Suppose that $(F, B)$ is a compact subset of $\tilde{G}$ over the soft regular topological space $(G, \tau, M)$. $U$ is an open soft neighborhood of $(F, B)$, for all $x \in(F, B)$, there is an open neighborhood of $V_{x}$ of $x$ such that $V_{x} \tilde{\subset} U$, and the collection $\left\{V_{x} \mid x \in(F, B)\right\}$ is an open cover of $(F, B)$, then there has a finite sub-collection $\left\{V_{x_{1}}, V_{x_{2}}, \cdots, V_{x_{n}}\right\}$ of $U_{x}$ which covers $(F, B)$, Assume $V=\bigcup_{i=1}^{n} V_{x_{i}}$, then $V$ is an open soft neighborhood of $(F, B)$, and $\bar{V}=\overline{\left(\bigcup_{i=1}^{n} V_{x_{i}}\right)}=\bigcup_{i=1}^{n} \overline{V_{x_{i}}} \tilde{\subset} U$

\section{Conclusion}

In this paper, we define the compact soft topological space, give soft separation axioms of soft rough topological space, we study soft compactness and soft separation axioms in the soft topological space over the rough soft formal context $T=(G, M, R, F)$ and give their properties. That is, we investigate the compactness and separate axioms of topological space combing topological structure and soft sets with rough sets and formal context, some different types of hybrid models are presented, which is topology over the rough soft formal context, and we name it as the soft rough topology. That offers a new method and tool in data analysis. We also can discuss more topological properties, such as countable axioms over the rough soft formal context $T=(G, M, R, F)$.

\section{Acknowledgment}

This work is supported by QingHai 135 programme and Qinghai Science Foundation (Grant No.2013-Z-913) .

\section{References}

[1] Euler, Leonhard, Solutio problematis ad geometriam situs pertinentis,Commentarii Academiae Scientiarum Imperialis Petropolitanae, 1736,8:128-140

[2] M.A Armsrtong,Basic topology, The world book publishing company ,Springer,2008

[3] L. A. Zadeh, ¡ $^{\circ}$ Fuzzy sets, $¡ \pm$ Information and Computation, vol. 8, pp. 338-353, 1965

1968

[4] C. L. Chang, $i^{\circ}$ Fuzzy topological spaces, $i \pm$ Journal of Mathematical Analysis and Applications, vol. 24, pp. 182-190, $89-103,1985$

[5] A. P. $\breve{s}$ ostak, On a fuzzy topological structure, Rendiconti del Circolo Matematico di Palermo. Serie II, no. 11, pp.

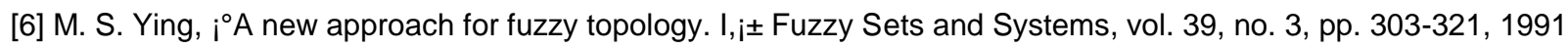

[7] Wille R (1982), Restructuring lattice theory: an approach based on hierarchies of concepts. Reprint in: ICFCA '09: Proceedings of the 7th International Conference on Formal Concept Analysis, Berlin, Heidelberg, 2009, 314

[8] B.Ganter, R.Wille (1999),Formal concept analysis,Mathematical Foundations,Springer, Berlin

[9] Pawlak Z(1982), Rough sets, International Journal of Computer And Information Science , 1:341-345

[10] Pawlak Z(1991), Rough sets: Theoretical Aspects of Reasoning about Data, Kluwer Academic Publishers, Boston

[11] Kent RE (1994), Rough concept analysis. In: Ziarko WP (ed) Rough sets, Fuzzy sets and knowledge discovery. Springer, London, 248-255 
[12] D. Molodtsov (1999), Soft set theory—first results, Computers Math. Appl. 37(4/5):19-31

[13] P.K.Maji, R.Biswas and A.R.Roy(2003), Soft set theory, Computers Math. Appl.45:555-562

[14] M.Shabir, M.Naz(2011) On soft topological spaces. Computers and Mathematics with Applications, 61:1786-1799

[15] Naim Cagman, Serkan Karatas, Serdar Enginoglu (2011), Soft Toplogy, Computers and Mathematics with Applications,62:351-358

[16] Sabir Hussain,Bashir Ahmad(2011), Some properties of soft topological spaces. Computers and Mathematics with Applications,62:4058-4067 $18: 717-728$

[17] Zhaowen Li , Tu sheng Xie(2014) The relationship among soft sets, soft rough sets and topologies, Soft comput,

[18] Fuli(2013) Rough formal Context Based on the Soft Sets 10th International Conference on Fuzzy Systems and Knowledge Discovery (FSKD 2013):152-156

[19] Fuli, Liu Zhen(2009) Concept lattice based on the rough sets ,International Journal of Advanced Intelligence , 1(1):141-151

[20] Fuli(2014) Topological Structure of Rough Soft Formal Context International Journalof Computers and Technologies 12(6):3536-3545

[21] FuLi, LiuZhen, Topological Separation axioms of Soft Rough Formal Context International Journal of Computers System Science and Engineering ( in record) 